\title{
GESTIÓN, ORDENACIÓN Y PROTECCIÓN DE LAS LADERAS ATERRAZADAS EN LA COMUNIDAD VALENCIANA
}

\author{
Sabina Asins Velis \\ Departamento de Planificación Territorial. Centro de Investigaciones sobre Desertificación-CIDE \\ Joan Romero González \\ Instituto Interuniversitario de Desarrollo Local. Universitat de València
}

\section{RESUMEN}

Dada la configuración del relieve de la Comunidad Valenciana, históricamente, los agricultores han aplicado técnicas culturales que les han permitido aterrazar las laderas donde ubicar los campos de cultivo. En la actualidad, estos bancales dotan a la Comunidad Valenciana de un paisaje singular y muy frágil que necesita de urgente gestión, ordenación y protección. El Reglamento de Paisaje de la Comunidad Valenciana, aprobado en el año 2006, incluye estos espacios entre las áreas a considerar como condicionantes de proyecto, lo que les confiere una cierta protección. Sin embargo, el hecho de que no esté aprobado el Plan de Acción Territorial del Paisaje de la Comunidad Valenciana, ni por ello los mecanismos de financiación que deberán vincularse a este Plan, hace que su preservación, a excepción de algunas ayudas públicas puntuales, recaiga directamente en los agricultores, con bajos ingresos dependientes de estas áreas abancaladas, secanas en su mayoría. La novedad del Reglamento es que estos espacios abancalados podrán ser considerados en el futuro planeamiento territorial gracias a su inclusión en los Estudios de Paisaje, en los Estudios de Integración Paisajística, en los Catálogos de Paisaje y en los Programas de Paisaje.

Palabras clave: terrazas de cultivo, planeamiento territorial, políticas de paisaje.

\section{ABSTRACT}

Given the topography and relief features of the Comunidad Valenciana (Spain), historically, farmers have used techniques that have enabled them to make terraces on the steep slopes in order to plant crops. Currently, these terraces or 'bancales' give the Comunidad Valenciana a very special and fragile landscape which urgently needs management, planning, and protection. The Landscape Regulation of 2006 includes these areas as part of those to be considered in assessing projects, which does give them some protection. However, the Spatial Action Plan of the Comunidad Valenciana has not been approved yet, and that therefore neither have the financing mechanisms that 
should accompany it. This means that the preservation of these areas falls directly upon the farmers, who have low incomes, and depend upon these areas, usually dry-cropping.

The new aspect in this Regulation is that these terraced areas can in the future be considered in spatial planning thanks to their inclusion in Landscape Studies, Landscape Integration Studies, Landscape Catalogues, and in the Landscape Programmes.

Key Words: terraced fields, spatial planning, landscape policies.

\section{INTRODUCCIÓN}

En el año 2009, la superficie de suelo dedicado al cultivo en la Comunidad Valenciana era de 688.382 hectáreas, es decir un 29,60\% del total de su extensión (Conselleria de Agricultura, Pesca y Alimentación, 2010: cuadro 4.2). Debido a la configuración montañosa del territorio de esta Comunidad un elevado porcentaje de esas explotaciones agrarias se incluyen en las áreas de montaña, con la característica común de que los campos de cultivo se disponen históricamente en bancales (Torró, 2003, 2005, 2007; Asins, 2004, 2006, 2009; Giménez Font, 2007, 2008; Grau y Pérez, 2008; Pérez Borreda, 2010). Ello confiere unas particularidades específicas a la gestión de estos espacios, no exentos de dificultades. Dificultades que desde hace años vienen siendo reconocidas por las políticas públicas que han incluido estos territorios en las denominadas «zonas desfavorecidas de montaña». En la Comunidad Valenciana, a esta categoría se acogen un total de 45 municipios de la provincia de Alicante, 79 de la de Castellón y 33 de la provincia de Valencia. Sin embargo, si bien en todos estos municipios está presente la técnica del aterrazamiento de laderas para ubicar los campos de cultivo, en los listados no constan otras localidades de montaña de la Comunidad en los que esta técnica de conservación de suelo y agua está ampliamente extendida, como serían Moixent y Vallada, entre otros, en donde no se pueden solicitar las «ayudas de indemnización compensatoria para los agricultores en las zonas con dificultades naturales de la Comunitat Valenciana» (Agencia Valenciana de Fomento y Garantía Agraria, 2011: Anexo I).

Estos municipios comparten similares tendencias relacionadas con las nuevas funciones que se otorgan a los territoriales rurales, que se caracterizan en la actualidad por ser mucho más complejos y plurales (Romero y Farinós, 2007; Romero, 2009: 53-72). En este contexto, son de enorme interés los retos que se presentan para el desarrollo de políticas territoriales capaces de superar las tradicionales visiones sectoriales y segmentadas, teniendo en consideración la multifuncionalidad de estos territorios. Este concepto implica el reconocimiento a los agricultores, quienes, además de la producción de alimentos, prestan una amplia gama de servicios adicionales que deben ser fomentados en tanto representan un potencial para el desarrollo endógeno de las áreas rurales. El interés por los valores paisajísticos, naturales y culturales, los retos ambientales de la agricultura, el mantenimiento de asentamientos rurales vivos y la seguridad alimentaria ya figuraban desde hacía tiempo, de una forma más o menos desconectada, como elementos de la política agraria. El principal logro de la noción de multifuncionalidad es haber logrado estructurarlos en un marco teórico consistente (Pinto-Correia y Vos, 2004; Vanslembrouck y Van Huylenbroeck, 2005: 1-39; Mander et al., 2007).

El enfoque territorial y más horizontal que la nueva política da a la economía rural es multisectorial e integrado, con el objeto de proteger el patrimonio rural y diversificar las actividades de los territorios rurales que permitan crear nuevas fuentes de ingreso y de empleo. Las recientes orientaciones de la Política Agrícola Común-PAC (European Commission, 2003 y 2006) han reforzado aún más esta tendencia hacia una progresiva 
atención en favor del desarrollo rural y de la introducción de prácticas más respetuosas con el medio natural y la conservación del paisaje. Una evolución que guarda coherencia tanto con la creciente multifuncionalidad territorial, como con la creciente contestación ciudadana en relación con prácticas agropecuarias poco saludables e insostenibles y el creciente aprecio por los valores culturales e identitarios del territorio y el paisaje. Estos objetivos de la PAC se han visto reforzados en las revisiones publicadas en 2009 y 2010 (European Commission, 2009 y 2010).

De igual modo, la Estrategia Territorial Europea y la Agenda Territorial Europea integran plenamente las cuestiones ambientales no como un elemento sectorial más, sino como un elemento central del modelo de desarrollo territorial, lo que incrementa las posibilidades de implementación de políticas de uso del suelo (European Commission 1999, 2007 y 2011). Esta forma diferente de entender el territorio se ha trasladado también a la gestión de las zonas de cultivo, por lo que las áreas aterrazadas, objeto de nuestro estudio, se han visto beneficiadas por el desarrollo del concepto de «agricultura territorial», que ya se recogía en la normativa española desde el año 2007 - como por ejemplo en la ley para el desarrollo sostenible del medio rural (Ley 45/2007 y Ministerio de la Presidencia, 2010) - .

En líneas generales, el modelo agrícola europeo se ejemplifica por: una agricultura «industrial», relacionada básicamente con las exigencias de los mercados globales; una agricultura «comercial», centrada en la producción y elaboración de productos de calidad, y una agricultura «territorial», integrada por explotaciones familiares que poseen una dimensión económica insuficiente y se encuentran en áreas con limitaciones físicas o económicas o tienen un especial valor medioambiental (González Regidor 2000: 110; 2003: 176). Estos dos últimos tipos de agricultura, la comercial de productos de calidad, pero sobre todo la territorial, son de aplicación a los espacios aterrazados. El particular valor medioambiental de las áreas aterrazadas históricas constituye uno de los principales «bienes públicos» que la actividad agrícola presta en las áreas de montaña (Cooper et al., 2009: 215), lo que es de sumo interés a la hora de reforzar los enfoques territoriales de la agricultura para mantener activos esos territorios especialmente frágiles. Con ello, se potencia la relación entre agricultura, políticas de desarrollo rural, ordenación del paisaje y, en sentido amplio, las políticas de cohesión territorial, siguiendo las directrices europeas (Mata, 2010).

La riqueza del paisaje aterrazado, además, queda reforzada por ser claro reflejo del concepto de paisaje, tal y como lo define el Convenio Europeo del Paisaje al incidir en que «el carácter del paisaje es el resultado de la interacción dinámica de factores naturales y/o humanos» (Jefatura del Estado, 2008), interacción que no ha podido ser mayor en estos campos de cultivo aterrazados (Figuras 1 y 2 ).

Por ello, las políticas públicas deben tener por finalidad la gestión adecuada de estos paisajes. En este sentido, la Comunidad Valenciana se integra en el grupo de territorios pioneros en reconocer los valores de nuestros paisajes agrarios, pues su conservación, en sentido amplio, está ya contemplada en su Reglamento de Paisaje (Generalitat Valenciana, 2006), como se detalla en el capítulo II dedicado a las Normas de Integración Paisajística:

- Los elementos topográficos artificiales tradicionales significativos, tales como muros, bancales, senderos, caminos tradicionales, escorrentías, setos y otros análogos se incorporarán como condicionante de proyecto, conservando y resaltando aquellos que favorezcan la formación de un paisaje de calidad y proponiendo acciones de integración necesarias para aquellos que lo pudieran deteriorar. Las acciones de integración serán coherentes con las características y el uso de los elementos topográficos artificiales, garantizando la reposición de dichos elementos cuando resultaren afectados por la ejecución de cualquier tipo de obra (Capítulo II-Artículo 20-3). 


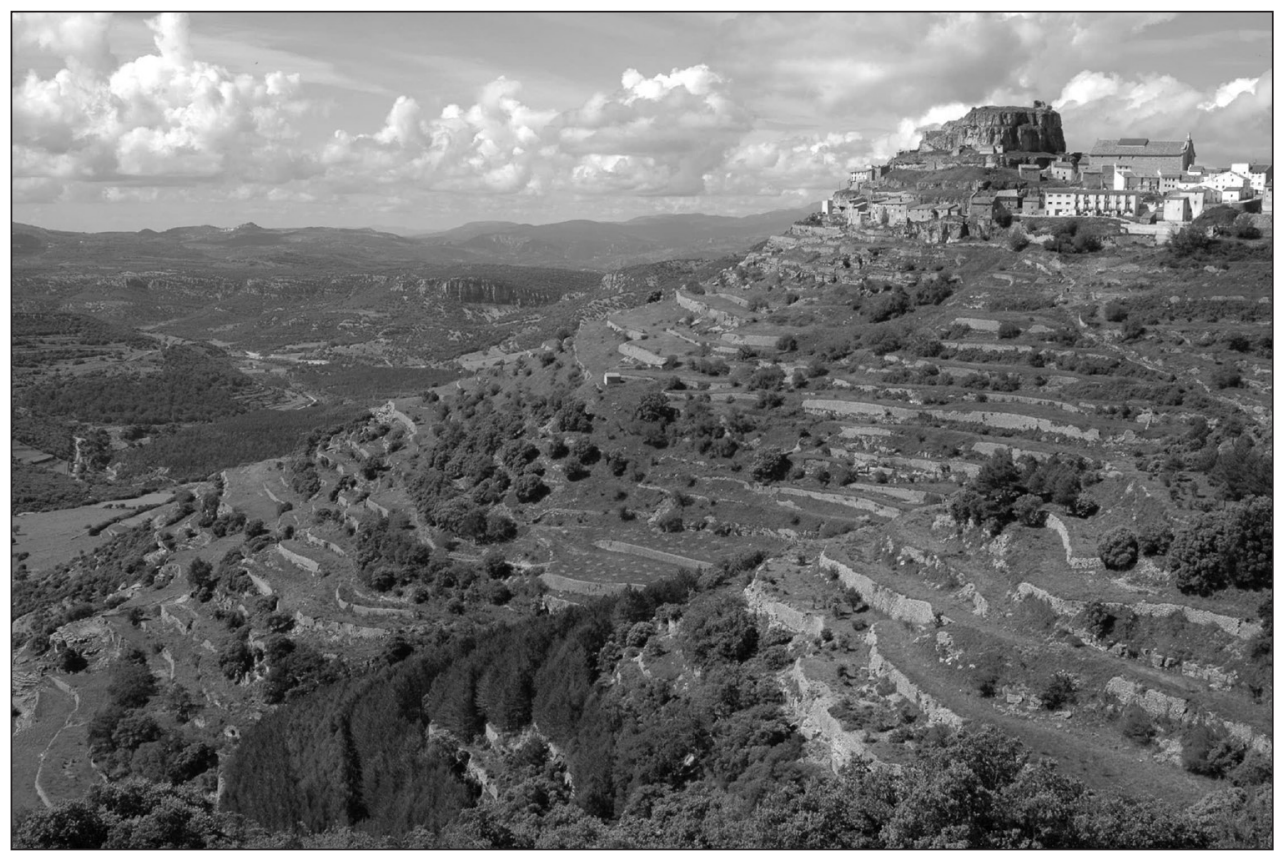

Figura 1. Aterrazamientos en Ares del Maestre (Castellón).

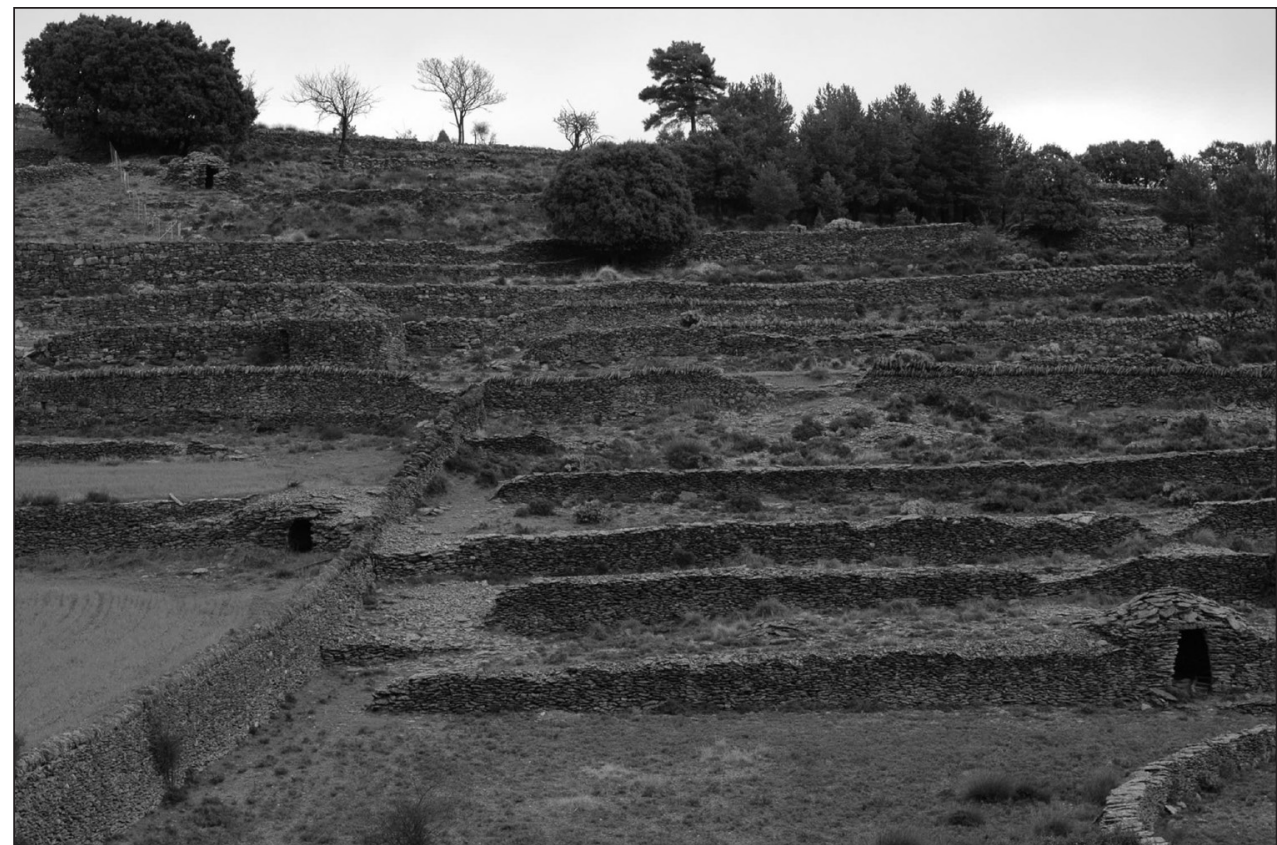

FIgURA 2. Terrazas de Vilafranca (Castellón). 
- En general, se mantendrá el paisaje agropecuario tradicional y característico de los espacios rurales por su contribución a la variedad del paisaje e integración en él de las áreas urbanizables previstas, permitiendo aquellos cambios que garanticen su integración paisajística (Capítulo II-Artículo 20-6).

Y no obstante estar reconocido en el citado Reglamento, la dificultad de la gestión de este paisaje construido a lo largo de los siglos radica en cómo integrarlo en los instrumentos de planificación territorial. Como base, el Artículo 6 del Reglamento especifica que los poderes públicos implementarán las políticas de paisaje mediante acciones que tengan por objeto la protección, gestión y ordenación de éste, puntualizando qué se entiende por cada una de estas acciones. Así distingue entre:

- Protección de los paisajes: comprende las actuaciones encaminadas a conservar y mantener los aspectos significativos o característicos de un paisaje, justificados por su valor patrimonial derivado de su configuración natural y/o la acción del hombre.

- Gestión de los paisajes: se entienden las actuaciones encaminadas, desde una perspectiva de desarrollo sostenible, a garantizar el mantenimiento regular de un paisaje, con el fin de guiar y armonizar las transformaciones inducidas por los procesos sociales, económicos y medioambientales.

- Ordenación de los paisajes: en relación a las actuaciones que presenten un carácter prospectivo particularmente acentuado con vistas a mejorar, restaurar o crear paisajes.

La finalidad de estas acciones es conservar y, en su caso, ordenar y gestionar los espacios, recursos y elementos naturales y culturales, para impedir la alteración o degradación de

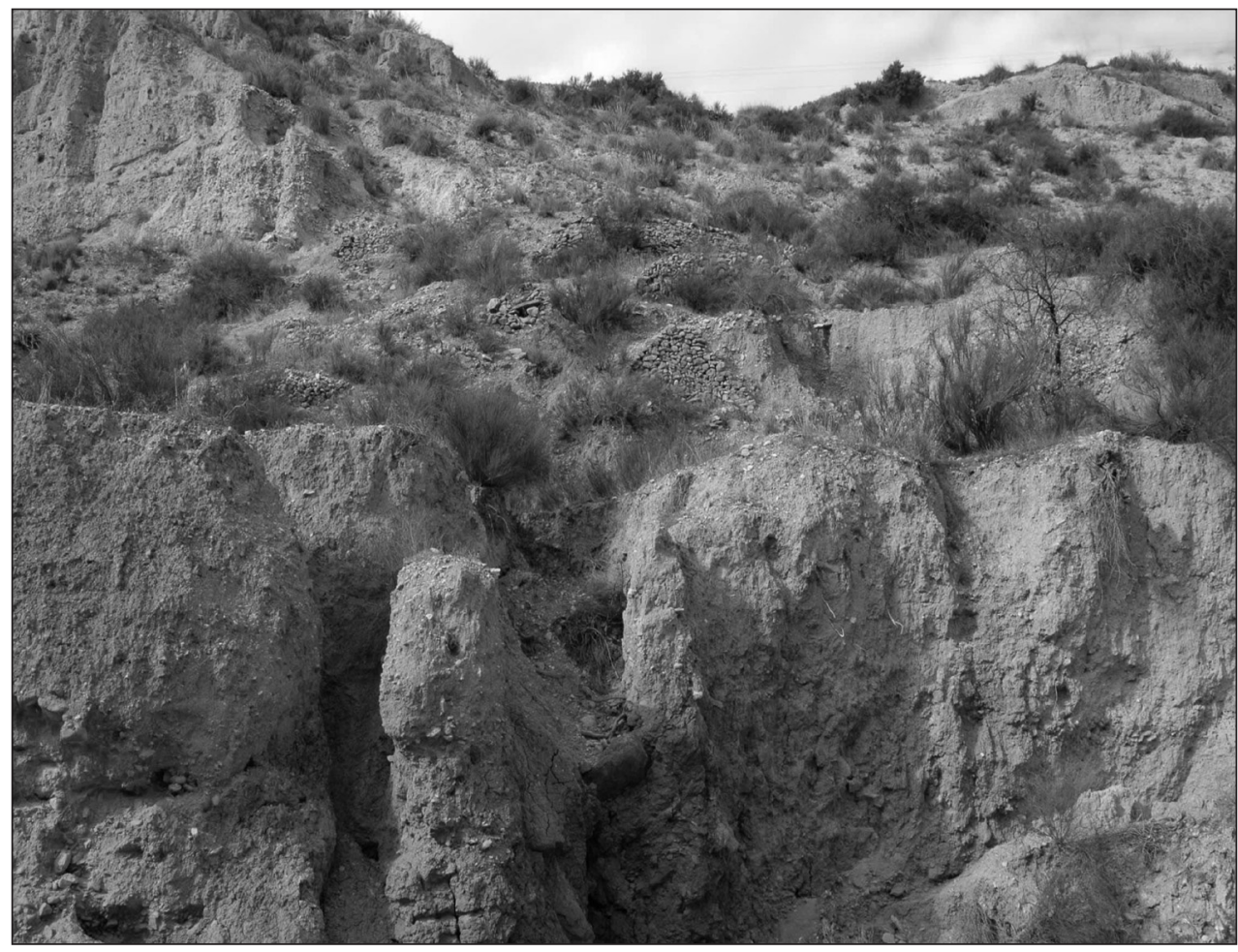

Figura 3. Procesos de erosión en campos de cultivo aterrazados abandonados (Petrer, Alicante). 
sus valores paisajísticos o propiciar su recuperación (Artículo 7a). Sin embargo, debemos recordar que la Generalitat Valenciana todavía no ha aprobado el Plan de Acción Territorial del Paisaje de la Comunidad Valenciana, y, de hecho, el trabajo de campo evidencia esa ausencia. Son numerosas las laderas aterrazadas que se encuentran en total estado de abandono; proceso que se sigue desde mediados del siglo XX. Entre los importantes riesgos que se asocian a ese proceso de abandono debemos destacar aquellos riesgos ambientales (como serían la erosión de las laderas por el desmoronamiento de los muros de mampostería - Figura 3-, la revegetación de áreas antiguamente abancaladas, sin prácticas de limpieza del bajo monte que incrementa el riesgo de incendios forestales, la pérdida de biodiversidad por la disminución del número de especies de fauna y flora asociadas a los campos de cultivo) económicos (emigración por disminución de renta) y socio-culturales (abandono de minas de agua, balsas, aljibes, pérdida de identidad local, etc) que afectan a importantes áreas aterrazadas de este territorio (Lasanta et al., 2001 y 2005; AA.VV., 2007).

Por ello, el objetivo de este estudio es profundizar en los instrumentos de gestión, ordenación y protección de estos paisajes en la Comunidad Valenciana y evaluar la viabilidad de su inclusión en la planificación territorial, tanto en los planes territoriales parciales como en los planes directores territoriales.

\section{INSTRUMENTOS DE GESTIÓN, ORDENACIÓN Y PROTECCIÓN DEL PAI- SAJE ATERRAZADO EN LA COMUNIDAD VALENCIANA}

El paisaje mediterráneo está en continua transformación. Los factores inductores del cambio son debidos tanto a aspectos naturales como socioeconómicos, políticos, tecnológicos o culturales. Desde mediados del siglo XX se ha producido un importante abandono del espacio agrícola aterrazado del interior de la Comunidad Valenciana, lo que ha implicado el desmoronamiento de los muros de piedra seca y la ausencia de mantenimiento del rico patrimonio cultural asociado a los campos de cultivo. Estos elementos, si bien están contemplados en el Reglamento de Paisaje de la Comunidad Valenciana, presentan unas particularidades que hace difícil su protección. En general, se trata de espacios secanos, difícilmente mecanizables y alejados de los núcleos de población, a veces por accesos en muy mal estado. Pero lo más importante es que el mantenimiento de estos paisajes, en muchas ocasiones calificados de «escénicos» por su gran belleza, recae en los mismos agricultores, caracterizados la mayoría de las veces por las bajas rentas que obtienen de esos cultivos dispuestos en bancales, generalmente en explotaciones de pequeño tamaño.

Las funciones de estos paisajes se encuentran ampliamente reconocidas en las disposiciones y reglamentos europeos. Así se incluyen en el Plan de Acción de Biodiversidad para la Agricultura, en la Estrategia de Desarrollo Sostenible, en la Estrategia Temática de Protección del Suelo, en las medidas incluidas en el Plan de Desarrollo Rural 2007-2013, y sobre todo en los Espacios Agrarios de Alto Valor Natural (Figura 4) (European Commission, 2001, 2004, 2008; Council of the European Union, 2006; Commission of the European Communities, 2005, 2006; European Commission, 2005; Comisión de las Comunidades Europeas, 2006, European Environment Agency, UNEP Regional Office for Europe, 2004).

Por cuanto se refiere al territorio nacional y autonómico, el Real Decreto 2352/2004 y el Decreto 91/2006 (Ministerio de Agricultura, Pesca y Alimentación, 2004: Artículo 4; Conselleria de Agricultura, Pesca y Alimentación, 2006: Anexo II) exponen en los requisitos mínimos de las «buenas condiciones agrarias y medioambientales de la condicionalidad», aquellos requeridos para evitar la erosión en los campos de cultivo, entre los que se cita el mantenimiento de las terrazas de retención, estableciendo la obligación de mantenerlas en buen estado de conservación. 


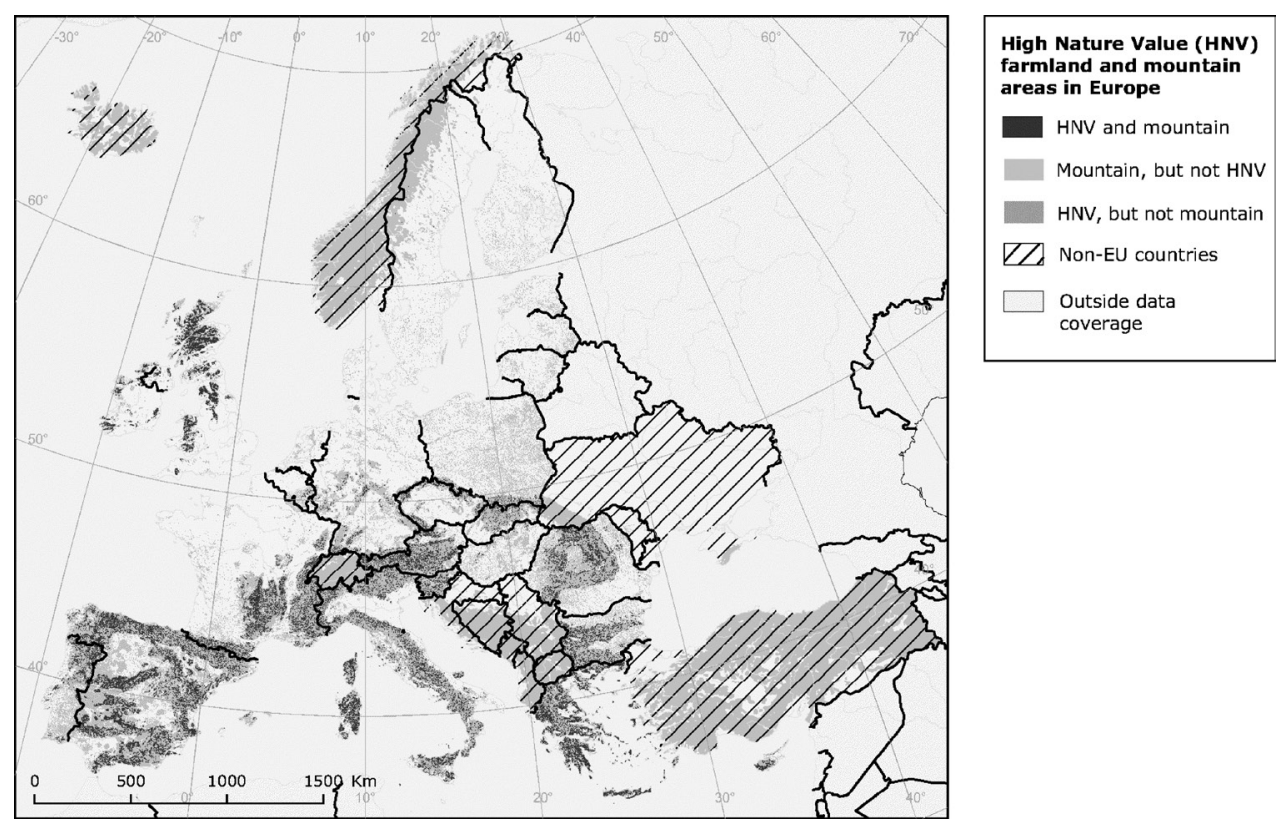

Figura 4. Explotaciones Agrícolas de Alto Valor Natural en Europa. En la Comunidad Valenciana coinciden con las áreas de montaña aterrazadas. Fuente: European Environment Agency, 2010: 6.

Dado que las competencias en materia de territorio están transferidas a las Comunidades Autónomas, la Generalitat Valenciana, con el fin de colaborar en el cumplimiento de esos requisitos, dispone de varias líneas presupuestarias para ayudar a compensar los costes adicionales a los que los agricultores deben hacer frente para mantener estas infraestructuras de conservación de suelo y agua (Tabla 1); algunas de esas partidas son a su vez cofinanciadas con los fondos FEADER y la Administración General del Estado.

De todas ellas, la más específica es la medida agroambiental de acompañamiento de la PAC denominada «lucha contra la erosión en cultivos leñosos en terrazas o bancales». Sin embargo, esta medida sigue siendo insuficiente, pues cubre únicamente determinados cultivos, como son el almendro, avellano, algarrobo y cerezo, en pendientes superiores al $8 \%$, y queda limitada a ciertos municipios, que no incluyen desde luego a todos aquellos en los que los aterrazamientos cumplen su función de conservación de suelo y agua. Los municipios incluidos son un total de 45 en la provincia de Alicante, 31 en la de Castellón y únicamente 3 municipios en la provincia de Valencia (Agencia Valenciana de Fomento y Garantía Agraria, 2011, Anexo III). En el año 2010 se tramitaron un total de 505 solicitudes al amparo de esta ayuda, que atendía a un total de 2.326 hectáreas, de ellas 1.408 ha dedicadas al cultivo del almendro, 636 ha al cerezo, 252 ha al avellano y únicamente 30 ha de algarrobos. Los demás cultivos leñosos, incluidos los olivos, están excluidos de estas ayudas. El total de la subvención alcanzó los 405.000 euros. Las ayudas agroambientales, en general, se co-financian en un 53,69\% por los fondos FEADER, en un 39,19\% por la Administración General del Estado y en un 7,12\% por la Generalitat Valenciana.

Si bien todas estas ayudas son de gran interés para preservar el paisaje aterrazado de la Comunidad Valenciana, se debería ir todavía un paso más allá, incluyendo a un mayor número de municipios y beneficiarios que pudieran acogerse a ellas. Además sería impor- 
Tabla 1

INSTRUMENTOS DE FINANCIACIÓN DE LOS COSTES SUPLEMENTARIOS A QUE DEBEN HACER FRENTE LOS AGRICULTORES PARA MANTENER EL PAISAJE ATERRAZADO EN LA COMUNIDAD VALENCIANA

\begin{tabular}{|c|c|}
\hline ORGANISMO CONVOCANTE & DENOMINACIÓN DE LA AYUDA \\
\hline \multirow[t]{3}{*}{$\begin{array}{l}\text { Conselleria de Agricultura, Pesca y } \\
\text { Alimentación }\end{array}$} & $\begin{array}{l}\text { Indemnización compensatoria a los agricultores } \\
\text { por las dificultades naturales en zonas de mon- } \\
\text { taña (en determinados municipios) }\end{array}$ \\
\hline & $\begin{array}{l}\text { Agroambientales: Lucha contra la erosión en } \\
\text { cultivos leñosos en terrazas o bancales (determi- } \\
\text { nados cultivos y municipios) }\end{array}$ \\
\hline & $\begin{array}{l}\text { RURALTER-Paisaje: restauración de construc- } \\
\text { ciones emblemáticas de piedra seca (municipios } \\
\text { de hasta } 40.000 \text { habitantes) }\end{array}$ \\
\hline $\begin{array}{l}\text { Conselleria de Infraestructuras, Te- } \\
\text { rritorio y Medio Ambiente }\end{array}$ & $\begin{array}{l}\text { Reconstrucción de muros de mampostería en } \\
\text { terrenos forestales y en una franja de seguridad } \\
\text { de } 500 \text { metros alrededor de los mismos, que se } \\
\text { subvencionan con } 170 \text { euros } / \mathrm{m}^{3} \text {, hasta un máxi- } \\
\text { mo de } 17.000 \text { euros }\end{array}$ \\
\hline $\begin{array}{l}\text { Conselleria de Turismo, Cultura y } \\
\text { Deporte (a los Ayuntamientos y enti- } \\
\text { dades sin ánimo de lucro, cofinancia- } \\
\text { ción por parte de estos con al menos } \\
\text { un } 25 \% \text { del importe de la actuación) }\end{array}$ & $\begin{array}{l}\text { Preservación del patrimonio de la piedra en } \\
\text { seco }\end{array}$ \\
\hline
\end{tabular}

tante que se revisaran los requisitos para poderlas solicitar (Generalitat Valenciana, 2010: 420-421, Agencia Valenciana de Fomento y Garantía Agraria, 2011), ya que son numerosos los campos abancalados que no alcanzan las 0,3 hectáreas requeridas como extensión mínima, y que incluso son cultivados por agricultores ya retirados, que no pueden acogerse a las ayudas pero que cumplen una importante función al conservar enclaves de pequeñas dimensiones pero muy valiosos a nivel ambiental y cultural.

Analizadas las vías actuales de financiación más importantes, nos gustaría centrarnos a continuación en cómo se incluyen los paisajes aterrazados en los Instrumentos para la Protección, Ordenación y Gestión del Paisaje, contemplados en la Ley de Ordenación del Territorio y Protección del Paisaje (Generalitat Valenciana, 2004). El Reglamento de Paisaje (Generalitat Valenciana, 2006) especifica que los Instrumentos diseñados para tal fin son los siguientes (Tabla 2): 
Tabla 2

INSTRUMENTOS PARA LA PROTECCIÓN, ORDENACIÓN Y GESTIÓN DEL PAISAJE DE LA COMUNIDAD VALENCIANA (GENERALITAT VALENCIANA, 2006)

\begin{tabular}{|l|}
\hline $\begin{array}{c}\text { INSTRUMENTOS PARA LA PROTECCIÓN, ORDENACIÓN Y GESTIÓN DEL PAISAJE DE LA } \\
\text { COMUNIDAD VALENCIANA }\end{array}$ \\
\hline Plan de Acción Territorial de Paisaje \\
\hline Estudios de Paisaje \\
\hline Estudios de Integración Paisajística \\
\hline Catálogos de Paisaje \\
\hline Programas de Paisaje \\
\hline
\end{tabular}

En su Título III se especifica que las funciones de estos Instrumentos se concretan en:

- contribuir a definir los objetivos para un desarrollo sostenible, donde coexista desarrollo y preservación de los valores paisajísticos;

- identificar los rasgos medioambientales, culturales y visuales de una localidad valorados por la población;

- definir la capacidad de un lugar para absorber el cambio y el desarrollo sin dañar los valores de su paisaje; y

- establecer las condiciones para que cualquier transformación del paisaje se realice con el objetivo de desarrollo sostenible y mejora de la calidad de vida.

En la actualidad, el Plan de Acción Territorial de Paisaje de la Comunidad Valenciana se encuentra en la fase de consultas de la versión preliminar. Tampoco se han publicado las Guías Metodológicas para la Valoración de Paisajes ni las Guías de Participación Pública en Paisaje. En su defecto, el Reglamento indica que los distintos municipios deben realizar los denominados Estudios de Paisaje, en los que se debe incluir la identificación y delimitación de Unidades de Paisaje y de los Recursos Paisajísticos. Una vez fijado el valor del paisaje, se podrán determinar los Objetivos de Calidad para cada uno de ellos, que se utilizarán para determinar desde su máxima protección hasta su necesaria redefinición. Estos estudios se deben realizar mediante la confección de un Catálogo, que sin duda debe incluir las particularidades agrícolas, naturales y culturales de los paisajes aterrazados (Figura 5).

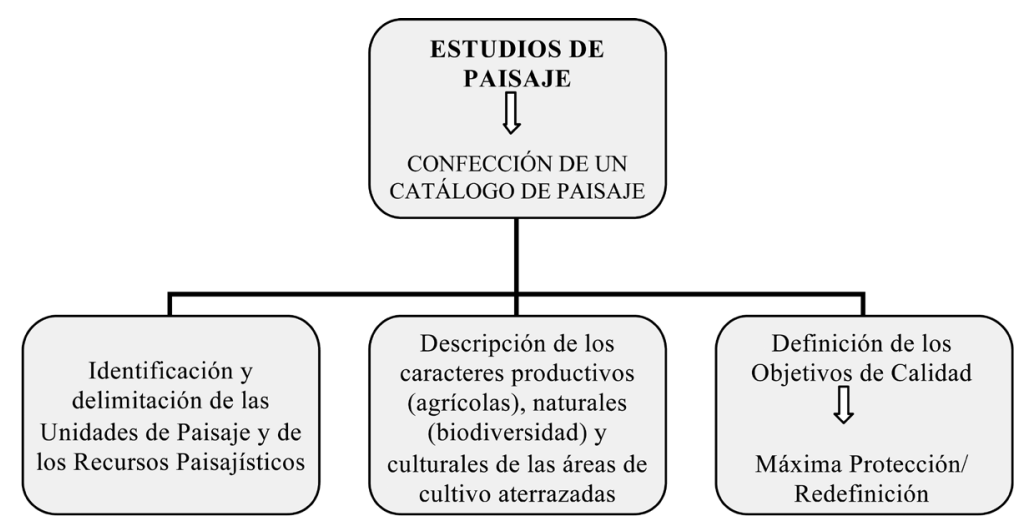

Figura 5. Inclusión de las áreas de cultivo aterrazadas en los Estudios de Paisaje, previstos en el Reglamento de Paisaje de la Comunidad Valenciana. 
Además cabe también la posibilidad de incluir estos paisajes en el Sistema de Espacios Abiertos (definidos en la Exposición de Motivos del Reglamento), que comprende aquel suelo clasificado como no urbanizable para el planeamiento urbanístico. De igual forma, podrían considerarse en los Sistemas de Asentamientos Urbanos, ya que aquí se incluyen las propuestas de estrategias de extensión, reforma o consolidación de los mismos (Nogué y Sala, 2006). No debemos olvidar que la ampliación de los asentamientos urbanos en la Comunidad Valenciana se hace en numerosas ocasiones en las zonas de huerta adyacentes a los núcleos urbanos, históricamente abancaladas - siguiendo un patrón muy difundido, como en Chelva, Bocairent, Petrer, etc. (Figura 6).

De igual modo, las áreas de cultivo aterrazadas se pueden incorporar a los Estudios de Integración Paisajística, cuyo objetivo, según el Reglamento citado, es analizar la incidencia de determinadas actuaciones en el paisaje y proponer las medidas correctoras y compensatorias de los impactos paisajísticos que hagan viable el proyecto. Estos Estudios deberían hacerse a escala supramunicipal, considerando sobre todo la escala de cuenca, e integrando políticas de carácter sectorial, con el objetivo de ser incorporados a los Planes Territoriales Parciales que pudieran redactarse, con atribuciones superiores al planeamiento urbanístico. El objetivo final de estos Estudios de Integración Paisajística es la inclusión de las políticas de paisaje en el planeamiento general.

Por último, los Programas de Paisaje, que según el Reglamento materializarán las acciones de gestión que se deriven de los Estudios y de las políticas de paisaje, deberían incluir una figura similar al Fondo para la Protección, Gestión y Ordenación del Paisaje de Cataluña, creado con la finalidad de destinarlo a actuaciones de mejora paisajística;

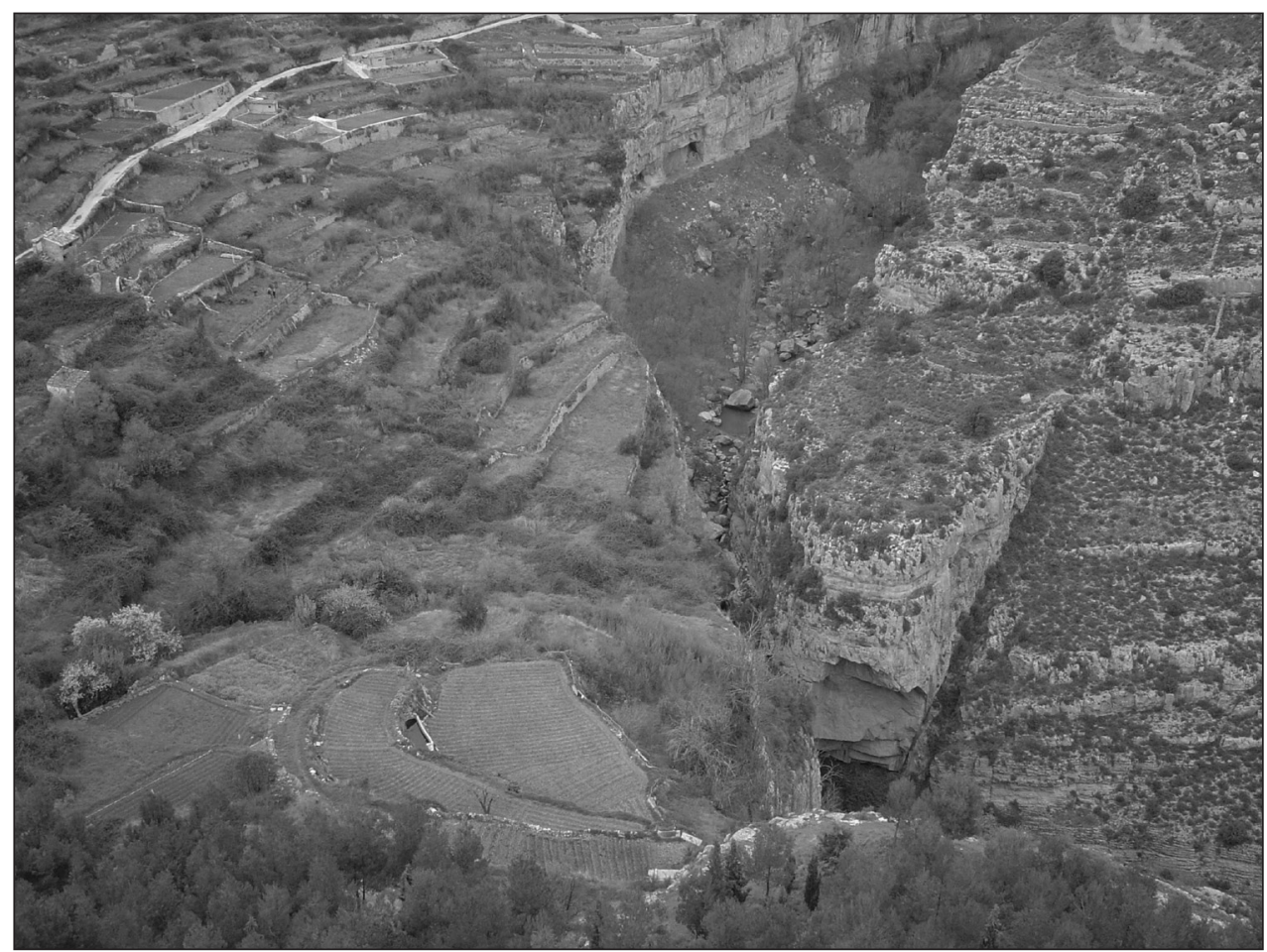

FIGURA 6. Parcelas de regadío aterrazadas junto al municipio de Alpuente (Valencia). 
fondo al que pueden optar tanto los entes públicos como las entidades privadas sin ánimo de lucro o los particulares (Adame, 2008). Este tipo de fondo sería de gran utilidad para compensar aquellas actuaciones que no pudieran ser cubiertas por las subvenciones ya en vigor, dependientes en su mayor parte de las directrices de la PAC.

\section{CONCLUSIONES}

En el año 2006, con la publicación del Reglamento de Paisaje de la Comunidad Valenciana, se abrió una nueva vía para la gestión, ordenación y protección de los paisajes agrarios aterrazados. Sin embargo, pasados cinco años, todavía no se ha aprobado el Plan de Acción Territorial del Paisaje, que se encuentra en fase de consultas de la versión preliminar. No obstante, los municipios tienen la oportunidad de incluir la presencia de esta rica técnica de conservación de suelo y agua en los Estudios de Paisaje, en los Estudios de Integración Paisajística y en los Catálogos de Paisaje. La complejidad de gestión de los paisajes aterrazados recomienda la imbricación de las políticas territoriales y sectoriales (sobre todo aquellas referidas a políticas agrarias, de desarrollo rural, de conservación de la naturaleza, de promoción turística asociada al paisaje, de infraestructuras, la política cultural y de educación, etc.) para lograr el máximo reconocimiento a una práctica mantenida por nuestros agricultores durante generaciones y que ha demostrado su eficacia en la conservación de unos recursos tan frágiles en territorios semi-áridos como el suelo y el agua. La inclusión de los paisajes aterrazados en el planeamiento general debe ir acompañada de mecanismos de financiación, complementarios a las ayudas ya previstas por la Generalitat Valenciana. Mecanismos que deberán alcanzar a un mayor número de beneficiarios de un mayor número de municipios de los que hasta ahora pueden optar a ellas, ya que hasta el momento son los agricultores valencianos los que están haciendo el mayor esfuerzo por dotarnos de unos espacios de tanta riqueza agrícola, ambiental y cultural.

Agradecimientos: Nuestro agradecimiento a D. Manuel Valdivia Benzal y a Dña. Teresa Velázquez, Jefa de Servicio de Gestión Agraria Sostenible de la Conselleria de Agricultura, Pesca y Alimentación.

\section{BIBLIOGRAFÍA}

AA.VV. (2007): Actes de les Jornades sobre Terrasses i Prevenció de Riscos Naturals. Consell Insular, Departament de Medi Ambient. Mallorca.

ADAME MARTÍNEZ, F.D. (2008): Instrumentos jurídicos de derecho civil y tributario para la protección y financiación del Monte Mediterráneo. Editorial Comares.

AGENCIA VALENCIANA DE FOMENTO Y GARANTÍA AGRARIA (2011): RESOLUCIÓN de 31 de enero de 2011, de la directora de la Agencia Valenciana de Fomento y Garantía Agraria, mediante la que se establece la convocatoria del procedimiento para la concesión de subvenciones a través de la solicitud única vinculadas al régimen de pago único, otras ayudas directas a la agricultura y la ganadería, ayudas agroambientales y de indemnización compensatoria para el año 2011. DOCV, núm. 6452, de 3 de febrero de 2011.

ASINS VELIS, S.; SÁNCHEZ DÍAZ, J. (2004): «Los aterrazamientos en la Comunidad Valenciana (España)», en La Cultura dei Terrazzamenti per la Salvaguardia del Paesaggio. Tecniche, risorse, strumenti. Atti del Convegno Internazionale. Edizione Menabó. Salerno, Italia, pp. 28-54. 
ASINS VELIS, S. (2006): «Linking historical Mediterranean terraces with water catchment, harvesting and distribution structures», en J.P. Morel, J.J. Tresserras Juan y J.C. Matamala (Eds.), The Archaeology of crop fields and gardens. Edipuglia. Bari, Italia, pp. 21-40.

ASINS VELIS, S. (2009): El paisaje agrario aterrazado: Diálogo entre el medio y el hombre en Petrer (Alicante). Colección: Desarrollo Territorial. Serie Estudios y Documentos, 5. Publicacions de la Universitat de València. Valencia; Research Centre on Traditional and Local Knowledge-IPOGEA, Italia.

COMISIÓN DE LAS COMUNIDADES EUROPEAS (2006): Decisión del Consejo de 20 de febrero de 2006 sobre las directrices estratégicas comunitarias de desarrollo rural (período de programación 2007-2013), (2006/144/CE), L55/20 Diario Oficial de la Unión Europea 25.2.2006.

COMMISSION OF THE EUROPEAN COMMUNITIES (2005): The 2005 Review of the EU Sustainable Development Strategy: Initial Stocktaking and Future Orientations. $\operatorname{COM}(2005) 37$ final.

COMMISSION OF THE EUROPEAN COMMUNITIES (2006): Brussels, 22.9.2006, COM(2006)231 final. Thematic Strategy for Soil Protection. [SEC(2006)620], [SEC(2006)1165].

CONSELLERIA DE AGRICULTURA, PESCA Y ALIMENTACIÓN (2006): DECRETO 91/2006, de 23 de junio, del Consell, sobre aplicación de la condicionalidad en relación con las ayudas directas en el marco de la Política Agrícola Común en el ámbito de la Comunitat Valenciana. DOCV, núm. 5291, de 29 de junio de 2006.

CONSELLERIA DE AGRICULTURA, PESCA Y ALIMENTACIÓN (2010): Publicaciones. Informes del Sector Agrario. Informe del Sector Agrario 2009, http://www.agricultura. gva.es

COOPER, T.; HART, K.; BALDOCK, D. (2009): The Provision of Public Goods Through Agriculture in the European Union, Report Prepared for DG Agriculture and Rural Development, Contract No 30-CE-0233091/00-28, Institute for European Environmental Policy. Londres.

COUNCIL OF THE EUROPEAN UNION (2006): Renewed EU Sustainable Development Strategy (EU SDS). Bruselas, 9 Junio 2006, 10117/06.

EUROPEAN COMMISSION (1999): ESDP-European Spatial Development Perspective, Towards Balanced and Sustainable Development of the Territory of the European Union, Luxembourg, Office for Official Publications of the European Communities.

EUROPEAN COMMISSION (2001): Biodiversity Action Plan for Agriculture. COM (2001) 162 final. Volume III.

EUROPEAN COMMISSION (2003): Reform of the Common Agricultural Policy, a longterm perspective for sustainable agriculture. Impact analysis. Directorate-General for Agriculture.

http://ec.europa.eu/agriculture/publi/reports/reformimpact/rep_en.pdf

EUROPEAN COMMISSION (2004): Environment and forestry. Biodiversity Action Plan for Agriculture: Implementation report.Directorate F. Horizontal aspects of rural development; SAPARD. F.1. Working document.

EUROPEAN COMMISSION (2005): Council Regulation (EC) 1698, On support for Rural Development by the EAFRD, 20-IX-2005.

EUROPEAN COMMISSION (2006): Council Decision of 20 February 2006 on Community strategic guidelines for rural development (programming period 2007 to 2013). (2006/144/EC).

http://eur-lex.europa.eu/LexUriServ/LexUriServ.do?uri=OJ:L:2006:055:0020:0029:EN:PDF

EUROPEAN COMMISSION (2007): Territorial Agenda of the European Union: Towards 
a more competitive and sustainable Europe of diverse Regions. Agreed on the occasion of the Informal Ministerial Meeting on Urban Development and Territorial Cohesion in Leipzig on 24 / 25 May 2007.

EUROPEAN COMMISSION (2008): The European Union's Biodiversity Action Plan: Halting the loss of biodiversity by 2010 - and beyond. Luxembourg: Office for Official Publications of the European Communities.

EUROPEAN COMMISSION (2009): Health Check of the CAP: current situation, Commission proposal and Council outcome. D.G. Agriculture and Rural Development. <http:// ec.europa.eu/agriculture/healthcheck/before_after_en.pdf $>$.

EUROPEAN COMMISSION (2010): Communication from the Commission to the European Parliament, the Council, the European Economic and Social Committee and the Committee of the Regions. The CAP towards 2020: Meeting the food, natural resources and territorial challenges of the future. $\operatorname{COM}(2010) 672$ final.

EUROPEAN COMMISSION (2011): Territorial Agenda of the European Union 2020. Towards an Inclusive, Smart and Sustainable Europe of Diverse Regions. Agreed at the Informal Ministerial Meeting of Ministers responsible for Spatial Planning and Territorial Development on 19th May 2011 Gödöllő, Hungary.

EUROPEAN ENVIRONMENT AGENCY, UNEP REGIONAL OFFICE FOR EUROPE (2004): High Nature Value Farmland: Characteristics, trends and policy challenges. EEA report, $\mathrm{n}^{\circ}$ 1, 2004. Luxembourg: Office for Official Publications of the European Communities.

EUROPEAN ENVIRONMENT AGENCY (2010): 10 messages for 2010 Mountain ecosystems Luxembourg: Office for Official Publications of the European Union.

GENERALITAT VALENCIANA (2004): Ley 4/2004 de 30 de junio, de la Generalitat, de Ordenación del Territorio y Protección del Paisaje. DOGV, núm. 4.788, de 2 de julio de 2004.

GENERALITAT VALENCIANA (2006): Conselleria de Territorio y Vivienda. Decreto 120/2006, de 11 de agosto, del Consell, por el que se aprueba el Reglamento de Paisaje de la Comunidad Valenciana. DOGV, núm. 5325, de 16 de agosto de 2006.

GENERALITAT VALENCIANA (2010): Programa de Desarrollo Rural de la Comunitat Valenciana cofinanciado por FEADER para el periodo 2007-2013; $2^{\text {a }}$ modificación aprobada por el C.S. de 17 de junio de 2010.

http://www.marm.es/es/desarrollo-rural/temas/periodo-de-programacion-2007-2013

GIMÉNEZ FONT, P. (2007): «Dinàmica del paisatge i terrasses de cultiu en la serra d'Aitana (Alacant). Claus interpretatives per al seu estudi», en V Congrés de la Institució Catalana d'Estudis Agraris. Barcelona.

GIMÉNEZ FONT, P. (2008): Las transformaciones del paisaje valenciano en el siglo XVIII: una perspectiva geográfica. Instituciò Alfons el Magnànim. Alacant.

GONZÁLEZ REGIDOR, J. (2000): El futuro del medio rural en España. Agricultura y desarrollo económico. Consejo Económico y Social. Madrid.

GONZÁLEZ REGIDOR, J. (2003): «Territorial Agriculture and Rural Development: From Agricultural Support to Territorial Policies», en The Future of Rural Policy: from sectoral to place-based policies in rural areas. Organisation for Economic Co-operation and Development (OCDE).

GRAU MIRA, I.; PÉREZ RODRÍGUEZ, V. (2008): «Aproximación arqueológica al estudio de los paisajes aterrazados en el área central valenciana». Lucentum XXVII, pp. 33-50.

JEFATURA DEL ESTADO (2008): Instrumento de Ratificación del Convenio Europeo del Paisaje (número 176 del Consejo de Europa), hecho en Florencia el 20 de octubre de 2000. BOE núm. 31, de 5 de febrero de 2008. 
LASANTA, T.; ARNÁEZ, J.; OSERÍN, M.; ORTIGOSA, L. (2001): «Marginal lands and erosion in terraced fields in the Mediterranean mountains: A case study in the Cameros Viejo (Northwestern Iberian System, Spain)». Mountain Research and Development, $\mathrm{n}^{\mathrm{o}} 21$ (1), pp. 69-76.

LASANTA, T.; VICENTE-SERRANO, S.M.; CUADRAT, J.M. (2005): «Mountain Mediterranean landscape evolution caused by the abandonment of traditional primary activities: a study of the Spanish Central Pyrenees». Applied Geography, n 25, pp. 47-65.

LEY 45/2007, de 13 de diciembre. Boletín Oficial del Estado ${ }^{\circ}$ 299, de 14 de diciembre de 2007.

MANDER, Ü.; WIGGERING, H.; HELMING, K. (Eds.) (2007): Multifunctional Land Use: Meeting Future Demands for Landscape Goods and Services. Springer. Berlín.

MATA OLMO, R. (2010): «La dimensión patrimonial del paisaje. Una mirada desde los espacios rurales», en Maderuelo, J. (Dir.), Paisaje y patrimonio. Abada Editores. Madrid, pp. 31-73.

MINISTERIO DE AGRICULTURA, PESCA Y ALIMENTACIÓN (2004): Real Decreto 2352/2004, de 23 de diciembre, sobre la aplicación de la condicionalidad en relación con las ayudas directas en el marco de la política agrícola común. Boletín Oficial del Estado $\mathrm{n}^{\circ}$ 309, de 24 de Diciembre de 2004.

MINISTERIO DE LA PRESIDENCIA (2010): Real Decreto 752/2010, de 4 de junio, por el que se aprueba el primer programa de desarrollo rural sostenible para el período 2010-2014 en aplicación de la Ley 45/2007, de 13 de diciembre, para el desarrollo sostenible del medio rural. Boletín Oficial del Estado n ${ }^{\circ} 142$, de 11 de junio de 2010.

NOGUÉ, J.; SALA, P. (2006): Prototipo de Catálogo de Paisaje: Bases conceptuales, Metodológicas y procedimentales para la elaboración de los Catálogos del Paisaje de Cataluña. Resumen del documento de referencia para los grupos de trabajo (edición revisada). Olot y Barcelona: Observatori del Paisatge. http://www.catpaisatge.net/fitxers/ resumenprototipo.pdf

PÉREZ BORREDA, J.R. (2010): Construyendo paisajes mediterráneos. Apropiación de tierras y transformaciones del paisaje en la Sierra de Enguera (1580-1930). Colección: Desarrollo Territorial. Serie Estudios y Documentos, 7. Publicacions de la Universitat de València. Valencia.

PINTO-CORREIA, T.; VOS, W. (2004): «Multifunctionality in Mediterranean landscapes - past and future», en The New Dimensions of the European Landscapes. Wageningen UR Frontis Series, Volume 4, pp. 135-164.

ROMERO GONZÁLEZ, J.; FARINÓS DASÍ, J. (2007): «Los territorios rurales en el cambio de siglo», en Romero, J. (Coord.), Geografía Humana: Procesos, riesgos e intertidumbres en un mundo globalizado. Ariel. Barcelona, pp. 355-383.

ROMERO GONZÁLEZ, J. (2009): Geopolítica y Gobierno del Territorio en España. Colección Crónica. Tirant lo Blanch. Valencia.

TORRÓ, J. (2003): «Arqueologia de la conquesta. Registre material, substitució de poblacions i transformació de l'espai rural valencià (segles XIII-XIV)», en M. Barceló, G. Feliu, A. Furió, M. Miquel, J. Sobrequés (Eds.), El feudalisme comptat i debatut: Formació i expansió del feudalisme català. Universitat de València. Valencia, pp. 153-200.

TORRÓ, J. (2005): «Terrasses irrigades a les muntanyes valencianes. Les transformacions de la colonització cristiana». Afers, $\mathrm{n}^{\circ}$ 51, pp. 301-356.

TORRÓ, J. (2007): «Vall de Laguar. Asentamientos, terrazas de cultivo e irrigación en las montañas de Šarq al-Andalus: un estudio local». Recerques del Museu d'Alcoi, no 16, pp. 151-182.

VANSLEMBROUCK, I.; VAN HUYLENBROECK, G. (2005): Landscape Amenities: Economic Assessment of Agricultural Landscapes. Springer. The Netherlands. 